九州大学学術情報リポジトリ

Kyushu University Institutional Repository

\title{
Matrix Trace Inequalities Related to Uncertainty Principle
}

Kosaki, Hideki

Faculty of Mathematics, Kyushu University

http://hdl. hand le. net/2324/11846

出版情報: International Journal of Mathematics. 16 (6)，pp.629-645，2005-07. World Scientific バージョン：

権利関係 : 


\title{
MHF Preprint Series
}

Kyushu University

21st Century COE Program

Development of Dynamic Mathematics with High Functionality

\section{Matrix trace inequalities related to uncertainty principle}

\author{
H. Kosaki
}

MHF 2005-1 1

( Received January 11, 2005 )

\author{
Faculty of Mathematics \\ Kyushu University \\ Fukuoka, JAPAN
}

\footnotetext{
${ }^{1}$ This paper is also published in Kyushu University Preprint Series in Mathematics as No. 2005-1.
} 


\title{
MATRIX TRACE INEQUALITIES RELATED TO UNCERTAINTY PRINCIPLE
}

\author{
HIDEKI KOSAKI
}

\begin{abstract}
In their recent article S. Luo and Z. Zhang conjectured the matrix trace inequality mentioned in the introduction below, which is motivated by uncertainty principle. We present a proof for the conjectured inequality.
\end{abstract}

\section{INTRODUCTION}

For a fixed density matrix $\rho$ the covariance

$$
\operatorname{Cov}_{\rho}(X, Y)=\operatorname{Tr}\left(\rho X Y^{*}\right)-\operatorname{Tr}(\rho X) \cdot \overline{\operatorname{Tr}(\rho Y)}
$$

gives rise to a positive sesquilinear form on the space of matrices. Heisenberg's uncertainty principle (in the matrix setting) states

$$
\frac{1}{4}|\operatorname{Tr}(\rho[A, B])|^{2} \leq \operatorname{Var}_{\rho}(A) \cdot \operatorname{Var}_{\rho}(B)
$$

for observables (i.e., self-adjoint matrices) $A, B$. Here, $\operatorname{Var}_{\rho}(A)\left(=\operatorname{Cov}_{\rho}(A, A)=\right.$ $\left.\operatorname{Tr}\left(\rho A^{2}\right)-(\operatorname{Tr}(\rho A))^{2}\right)$ is the variance of $A$ relative to $\rho$. This estimate is a consequence of the Cauchy-Schwarz inequality, and the left side here (i.e., the commutator part) arises from the imaginary part $\frac{1}{2 i} \operatorname{Tr}(\rho[A, B])$ of the $\operatorname{covariance}^{\operatorname{Cov}_{\rho}}(A, B)$. The real part can be expressed in terms of a certain anti-commutator, and a stronger estimate including its effect (known as Schrödinger's uncertainty principle) is also possible (see $\S 2$, especially Remark 2). On the other hand,

$$
\operatorname{Corr}_{\rho}(A, B)=\operatorname{Tr}(\rho A B)-\operatorname{Tr}\left(\rho^{1 / 2} A \rho^{1 / 2} B\right)
$$

is called the Wigner-Yanase correlation ([7]), and the (Wigner-Yanase) skew information $\mathrm{I}(\rho, A)=\operatorname{Corr}_{\rho}(A, A)\left(=-\frac{1}{2} \operatorname{Tr}\left(\left[\rho^{1 / 2}, A\right]^{2}\right)\right)$ has been investigated by many authors as a certain measurement of information content of $\rho$ relative to $A$. Quite a thorough account on these and related subjects can be found in [6].

In the recent article [5] S. Luo and Z. Zhang discussed various aspects of quantum measurement and the above-mentioned uncertainty principle with strong emphasis on the use of the skew information $\mathrm{I}(\rho, A)$. Among other things the inequality

$$
\frac{1}{4}|\operatorname{Tr}(\rho[A, B])|^{2}+\frac{1}{16}|\mathrm{I}(\rho, A+B)-\mathrm{I}(\rho, A-B)|^{2} \leq \mathrm{I}(\rho, A) \cdot \mathrm{I}(\rho, B)
$$

1991 Mathematics Subject Classification. 15A45; Secondary 47A30, 47A50, 81P15.

Key words and phrases. trace inequality, uncertainty principle, Wigner-Yanase skew information. 
was claimed as a main result in their article (see [5, Theorem 2 in p.1571]), which is equivalent to a certain Cauchy-Schwarz type estimate (see $\S 2$ and $\S 4.1$ for details). Motivated by this claim, they conjectured

$$
\begin{aligned}
\operatorname{Var}_{\rho} A \cdot \operatorname{Var}_{\rho} B- & \frac{1}{4}\left|\operatorname{Cov}_{\rho}(A, B)+\operatorname{Cov}_{\rho}(B, A)\right|^{2} \\
& \geq \mathrm{I}(\rho, A) \cdot \mathrm{I}(\rho, B)-\frac{1}{4}\left|\operatorname{Corr}_{\rho}(A, B)+\operatorname{Corr}_{\rho}(B, A)\right|^{2}
\end{aligned}
$$

(see [5, p.1572]), and its validity for $2 \times 2$ matrices and some other cases was checked. The conjecture means that the claimed inequality is stronger than Schrödinger's uncertainty principle in the sense that the former (together with the conjectured inequality) implies the latter.

The purpose of the article is to present a proof for their conjecture (i.e., the above second inequality). More generally we will deal with the famous one-parameter family $\{\mathrm{I}(\rho, A ; \alpha)\}_{0<\alpha<1}$ introduced by Dyson: $\mathrm{I}(\rho, A ; \alpha)=-\frac{1}{2} \operatorname{Tr}\left(\left[\rho^{\alpha}, A\right]\left[\rho^{1-\alpha}, A\right]\right)$. Our main result (Theorem 5 in $\S 3$ ) actually covers this family, and we will also clarify the equality condition for the inequalities in question (Proposition 6). In $\S 4.1$ we will point out that the above first inequality (i.e., the one claimed as [5, Theorem 2] by S. Luo and Z. Zhang) is unfortunately false.

The author would like to thank H. Araki for informing him of the conjecture studied in the article and also F. Hiai for discussions on presented materials.

\section{NOTATIONS AND PRELIMINARIES}

We will denote the set of all $n \times n$ matrices (resp. all $n \times n$ self-adjoint matrices) by $M_{n}(\mathbf{C})$ (resp. $M_{n}(\mathbf{C})_{s a}$ ). General matrices will be denoted by $X, Y, \cdots$ while letters $A, B, \cdots$ will be used to express self-adjoint ones. We assume that $\rho \in M_{n}(\mathbf{C})$ is a (distinguished) density matrix, i.e., a positive matrix satisfying $\operatorname{Tr}(\rho)=1$.

Lemma 1. We assume $0<\alpha<1$.

(i) We have

$$
|\operatorname{Tr}(\rho X)|^{2} \leq \operatorname{Tr}\left(\rho^{\alpha} X \rho^{1-\alpha} X^{*}\right), X \in M_{n}(\mathbf{C}) .
$$

In particular, $A \in M_{n}(\mathbf{C})_{\text {sa }}$ satisfies $(\operatorname{Tr}(\rho A))^{2} \leq \operatorname{Tr}\left(\rho^{\alpha} A \rho^{1-\alpha} A\right)$.

(ii) A normal matrix $X \in M_{n}(\mathbf{C})$ satisfies

$$
\operatorname{Tr}\left(\rho^{\alpha} X \rho^{1-\alpha} X^{*}\right) \leq \operatorname{Tr}\left(\rho|X|^{2}\right),
$$

and hence $A \in M_{n}(\mathbf{C})_{\text {sa }}$ satisfies $\operatorname{Tr}\left(\rho^{\alpha} A \rho^{1-\alpha} A\right) \leq \operatorname{Tr}\left(\rho A^{2}\right)$.

Proof. Let us consider the map

$$
(X, Y) \in M_{n}(\mathbf{C}) \times M_{n}(\mathbf{C}) \rightarrow \operatorname{Tr}\left(\rho^{\alpha} X \rho^{1-\alpha} Y^{*}\right) \in \mathbf{C} .
$$

Since

$$
\overline{\operatorname{Tr}\left(\rho^{\alpha} Y \rho^{1-\alpha} X^{*}\right)}=\operatorname{Tr}\left(X \rho^{1-\alpha} Y^{*} \rho^{\alpha}\right)=\operatorname{Tr}\left(\rho^{\alpha} X \rho^{1-\alpha} Y^{*}\right),
$$


the above gives rise to a sesquilinear form. It is also positive, i.e., $\operatorname{Tr}\left(\rho^{\alpha} X \rho^{1-\alpha} X^{*}\right) \geq$ 0, and the Cauchy-Schwarz inequality tells

$$
|\operatorname{Tr}(\rho X)|^{2}=\left|\operatorname{Tr}\left(\rho^{\alpha} X \rho^{1-\alpha} 1^{*}\right)\right|^{2} \leq \operatorname{Tr}\left(\rho^{\alpha} X \rho^{1-\alpha} X^{*}\right) \cdot \operatorname{Tr}\left(\rho^{\alpha} 1 \rho^{1-\alpha} 1^{*}\right),
$$

showing (i) because of $\operatorname{Tr}(\rho)=1$.

To show (ii), we consider the bounded continuous function $f(z)=\operatorname{Tr}\left(\rho^{z} X \rho^{1-z} X^{*}\right)$ on the strip $0 \leq \Re z \leq 1$, which is analytic on the interior. Boundary values admit the following upper bounds:

$$
\begin{aligned}
|f(i t)| & =\left|\operatorname{Tr}\left(\rho^{i t} X \rho^{1 / 2} \rho^{-i t} \rho^{1 / 2} X^{*}\right)\right| \\
& \leq\left\|\rho^{i t} X \rho^{1 / 2} \rho^{-i t}\right\|_{H S}\left\|X \rho^{1 / 2}\right\|_{H S}=\left\|X \rho^{1 / 2}\right\|_{H S}^{2}, \\
|f(1+i t)| & =\left|\operatorname{Tr}\left(\rho^{i t} \rho X \rho^{-i t} X^{*}\right)\right|=\left|\operatorname{Tr}\left(\rho^{i t} \rho^{1 / 2} X \rho^{-i t} X^{*} \rho^{1 / 2}\right)\right| \\
& \leq\left\|\rho^{i t} \rho^{1 / 2} X \rho^{-i t}\right\|_{H S}\left\|\rho^{1 / 2} X\right\|_{H S}=\left\|\rho^{1 / 2} X\right\|_{H S}^{2} .
\end{aligned}
$$

Here, $\|\cdot\|_{H S}$ means the Hilbert-Schmidt norm and $t \in \mathbf{R}$. The normality of $X$ implies $\left\|X \rho^{1 / 2}\right\|_{H S}^{2}=\left\|\rho^{1 / 2} X\right\|_{H S}^{2}=\operatorname{Tr}\left(\rho|X|^{2}\right)$ so that the desired estimate follows from the maximum modulus principle.

The estimate in Lemma 1,(ii) means $\left\|\rho^{\alpha / 2} X \rho^{(1-\alpha) / 2}\right\|_{H S} \leq\left\|\rho^{1 / 2} X\right\|_{H S}$. Extensive investigation on more general estimates in this nature (for general unitarily invariant norms) was carried out in [2].

For a fixed $\alpha \in(0,1)$ and $X, Y \in M_{n}(\mathbf{C})$ we set

$$
\operatorname{Corr}_{\rho}(X, Y ; \alpha)=\operatorname{Tr}\left(\rho X Y^{*}\right)-\operatorname{Tr}\left(\rho^{\alpha} X \rho^{1-\alpha} Y^{*}\right) \text {, correlation. }
$$

Hence, for $A, B \in M_{n}(\mathbf{C})_{s a}$ we have

$$
\begin{aligned}
& \operatorname{Corr}_{\rho}(A, B ; \alpha)=\operatorname{Tr}(\rho A B)-\operatorname{Tr}\left(\rho^{\alpha} A \rho^{1-\alpha} B\right), \\
& \operatorname{Corr}_{\rho}(A, A ; \alpha)=-\frac{1}{2} \operatorname{Tr}\left(\left[\rho^{\alpha}, A\right]\left[\rho^{1-\alpha}, A\right]\right) .
\end{aligned}
$$

In the literature (see $[1,6,7]$ for instance) the quantities

$$
\mathrm{I}(\rho, A ; 1 / 2)=\operatorname{Corr}_{\rho}(A, A ; 1 / 2)
$$

and $\operatorname{Corr}_{\rho}(A, B ; 1 / 2)$ are often called the Wigner-Yanase skew information and correlation, and the former is regarded as a certain measurement of information content of the density $\rho$ with respect to $A$. Its generalization to

$$
\mathrm{I}(\rho, A ; \alpha)=\operatorname{Corr}_{\rho}(A, A ; \alpha)(\geq 0)
$$

was suggested by Dyson, and the convexity of $\mathrm{I}(\rho, A ; \alpha)$ as a function of $\rho$ was established in the celebrated work [4] by Lieb. We will denote $\operatorname{Corr}_{\rho}(A, B ; 1 / 2)$ and $\mathrm{I}(\rho, A ; 1 / 2)$ simply by $\operatorname{Corr}_{\rho}(A, B)$ and $\mathrm{I}(\rho, A)$ respectively.

We have just observed $\operatorname{Corr}_{\rho}(X, X ; \alpha) \geq 0$ for $X$ normal (Lemma 1,(ii)), but let us emphasize that the sesquilinear form

$$
(X, Y) \in M_{n}(\mathbf{C}) \times M_{n}(\mathbf{C}) \rightarrow \operatorname{Corr}_{\rho}(X, Y ; \alpha) \in \mathbf{C}
$$


is not positive, i.e., $\operatorname{Corr}_{\rho}(X, X ; \alpha) \nsupseteq 0$. Indeed, for

$$
\rho=\left[\begin{array}{cc}
\lambda_{1} & 0 \\
0 & \lambda_{2}
\end{array}\right], \quad X=\left[\begin{array}{ll}
0 & a \\
b & 0
\end{array}\right]
$$

we easily compute

$$
\operatorname{Corr}_{\rho}(X, X ; \alpha)=\lambda_{1}^{\alpha}\left(\lambda_{1}^{1-\alpha}-\lambda_{2}^{1-\alpha}\right)|a|^{2}+\lambda_{2}^{\alpha}\left(\lambda_{2}^{1-\alpha}-\lambda_{1}^{1-\alpha}\right)|b|^{2}
$$

(which is negative for either $(a, b)=(1,0)$ or $(0,1)$ as long as $\left.\lambda_{1} \neq \lambda_{2}\right)$. On the other hand, although the quantity $\operatorname{Corr}_{\rho}(A, B ; \alpha)$ may not be real, the correspondence

$$
(A, B) \in M_{n}(\mathbf{C})_{s a} \times M_{n}(\mathbf{C})_{s a} \rightarrow \Re \operatorname{Corr}_{\rho}(A, B ; \alpha) \in \mathbf{R}
$$

certainly gives rise to a positive bilinear form on the real vector space $M_{n}(\mathbf{C})_{s a}$ so that the Cauchy-Schwarz inequality yields

$$
\left(\Re \operatorname{Corr}_{\rho}(A, B ; \alpha)\right)^{2} \leq \mathrm{I}(\rho, A ; \alpha) \cdot \mathrm{I}(\rho, B ; \alpha) .
$$

S. Luo and Z. Zhang derived the inequality mentioned in $\S 1$ (i.e., [5, Theorem 2]) from the stronger estimate $\left|\operatorname{Corr}_{\rho}(A, B)\right|^{2} \leq \mathrm{I}(\rho, A) \cdot \mathrm{I}(\rho, B)$ (for $\left.\alpha=1 / 2\right)$. However, (1) is not a positive sesquilinear form on the vector space $M_{n}(\mathbf{C})$ and the author sees no a priori reason for the validity of such a strong Cauchy-Schwarz type estimate (on the absolute value). The above (non-) positivity seems to be the point overlooked in [5], and [5, Theorem 2] (or equivalently, the above estimate on the absolute value) is indeed false as will be clarified in $§ 4.1$.

Remark 2. The correspondence

$$
(X, Y) \in M_{n}(\mathbf{C}) \times M_{n}(\mathbf{C}) \rightarrow\langle X, Y\rangle=\operatorname{Tr}\left(\rho X Y^{*}\right)-\operatorname{Tr}(\rho X) \cdot \overline{\operatorname{Tr}(\rho Y)} \in \mathbf{C}
$$

is a positive sesquilinear form. Indeed, the positivity is a consequence of the CauchySchwarz inequality (applied to $\left.(X, Y) \rightarrow \operatorname{Tr}\left(\rho X Y^{*}\right)\right)$ :

$$
|\operatorname{Tr}(\rho X)|^{2}=\left|\operatorname{Tr}\left(\rho X 1^{*}\right)\right|^{2} \leq \operatorname{Tr}\left(\rho X X^{*}\right) \cdot \operatorname{Tr}\left(\rho 11^{*}\right)=\operatorname{Tr}\left(\rho X X^{*}\right) .
$$

For $A, B \in M_{n}(\mathbf{C})_{s a}$ we set

$$
\begin{aligned}
& \operatorname{Cov}_{\rho}(A, B)=\langle A, B\rangle \quad(=\operatorname{Tr}(\rho A B)-\operatorname{Tr}(\rho A) \cdot \operatorname{Tr}(\rho B)), \text { covariance, } \\
& \operatorname{Var}_{\rho} A=\langle A, A\rangle \quad\left(=\operatorname{Tr}\left(\rho A^{2}\right)-(\operatorname{Tr}(\rho A))^{2}\right), \text { variance. }
\end{aligned}
$$

Lemma 1,(i) means

$$
\operatorname{Var}_{\rho}(A) \geq I(\rho, A ; \alpha)
$$

(see [5, Theorem 1]), and the Cauchy-Schwarz inequality (applied to $\langle\cdot, \cdot\rangle$ ) tells

$$
\left|\operatorname{Cov}_{\rho}(A, B)\right|^{2} \leq \operatorname{Var}_{\rho} A \cdot \operatorname{Var}_{\rho} B
$$


The product $\operatorname{Tr}(\rho A) \cdot \operatorname{Tr}(\rho B)$ being real, we have

$$
\begin{aligned}
\Re \operatorname{Cov}_{\rho}(A, B) & =\Re \operatorname{Tr}(\rho A B)-\operatorname{Tr}(\rho A) \cdot \operatorname{Tr}(\rho B) \\
& =\frac{1}{2}(\operatorname{Tr}(\rho A B)+\operatorname{Tr}(\rho B A)-2 \operatorname{Tr}(\rho A) \cdot \operatorname{Tr}(\rho B)), \\
\Im \operatorname{Cov}_{\rho}(A, B) & =\Im \operatorname{Tr}(\rho A B)=\frac{1}{2 i}(\operatorname{Tr}(\rho A B)-\operatorname{Tr}(\rho B A)) .
\end{aligned}
$$

By setting $A_{0}=A-\operatorname{Tr}(\rho A) 1, B_{0}=B-\operatorname{Tr}(\rho B) 1$, we easily observe

$$
\operatorname{Tr}(\rho A B)+\operatorname{Tr}(\rho B A)-2 \operatorname{Tr}(\rho A) \cdot \operatorname{Tr}(\rho B)=\operatorname{Tr}\left(\rho\left\{A_{0}, B_{0}\right\}\right)
$$

with the anti-commutator $\left\{A_{0}, B_{0}\right\}=A_{0} B_{0}+B_{0} A_{0}$ so that (3) means

$$
\frac{1}{4}\left|\operatorname{Tr}\left(\rho\left\{A_{0}, B_{0}\right\}\right)\right|^{2}+\frac{1}{4}|\operatorname{Tr}(\rho[A, B])|^{2} \leq \operatorname{Var}_{\rho} A \cdot \operatorname{Var}_{\rho} B .
$$

This inequality is known as Schrödinger's uncertainty principle whereas the weaker (commutator) estimate

$$
\frac{1}{4}|\operatorname{Tr}(\rho[A, B])|^{2} \leq \operatorname{Var}_{\rho} A \cdot \operatorname{Var}_{\rho} B
$$

is referred to as Heisenberg's uncertainty principle in the literature.

\section{MAIN RESULT}

In [5] the inequality

$$
\begin{aligned}
\operatorname{Var}_{\rho} A \cdot \operatorname{Var}_{\rho} B- & \frac{1}{4}\left|\operatorname{Cov}_{\rho}(A, B)+\operatorname{Cov}_{\rho}(B, A)\right|^{2} \\
& \geq \mathrm{I}(\rho, A) \cdot \mathrm{I}(\rho, B)-\frac{1}{4}\left|\operatorname{Corr}_{\rho}(A, B)+\operatorname{Corr}_{\rho}(B, A)\right|^{2}
\end{aligned}
$$

was conjectured (and its validity was checked for $2 \times 2$ matrices and some other cases). We prove the conjectured inequality (4) in this section. We will actually obtain a one-parameter version involving quantities such as $\mathrm{I}(\cdot ; \alpha)$ and $\operatorname{Corr}_{\rho}(\cdot, \cdot ; \alpha)$, $\alpha \in(0,1)$.

We may and do assume that the density matrix $\rho$ is diagonalized so that in the rest we will assume

$$
\rho=\operatorname{diag}\left(\lambda_{1}, \lambda_{2}, \cdots, \lambda_{n}\right)
$$

with $\lambda_{i}>0$. The general case $\lambda_{i} \geq 0$ can be obtained by the limiting argument thanks to the obvious continuity of relevant quantities. We begin by expressing these quantities in terms of matrix components.

Lemma 3. For self-adjoint matrices $A=\left[a_{i j}\right], B=\left[b_{i j}\right] \in M_{n}(\mathbf{C})_{s a}$ we have

$$
\Re \operatorname{Tr}(\rho A B)=\sum_{1 \leq i<j \leq n} \alpha_{i j} \Re\left(a_{i j} \overline{b_{i j}}\right)+\sum_{i=1}^{n} \lambda_{i} a_{i i} b_{i i} \text { with } \alpha_{i j}=\lambda_{i}+\lambda_{j},
$$

and

$\Re \operatorname{Tr}\left(\rho^{\alpha} A \rho^{1-\alpha} B\right)=\sum_{1 \leq i<j \leq n} \beta_{i j} \Re\left(a_{i j} \overline{b_{i j}}\right)+\sum_{i=1}^{n} \lambda_{i} a_{i i} b_{i i}$ with $\beta_{i j}=\lambda_{i}^{\alpha} \lambda_{j}^{1-\alpha}+\lambda_{i}^{1-\alpha} \lambda_{j}^{\alpha}$. 
In particular, with $A=B$ we have

$$
\begin{aligned}
\operatorname{Tr}\left(\rho A^{2}\right) & =\sum_{i<j} \alpha_{i j}\left|a_{i j}\right|^{2}+\sum_{i} \lambda_{i} a_{i i}^{2}, \\
\operatorname{Tr}\left(\rho B^{2}\right) & =\sum_{i<j} \alpha_{i j}\left|b_{i j}\right|^{2}+\sum_{i} \lambda_{i} b_{i i}^{2}, \\
\operatorname{Tr}\left(\rho^{\alpha} A \rho^{1-\alpha} A\right) & =\sum_{i<j} \beta_{i j}\left|a_{i j}\right|^{2}+\sum_{i} \lambda_{i} a_{i i}^{2}, \\
\operatorname{Tr}\left(\rho^{\alpha} B \rho^{1-\alpha} B\right) & =\sum_{i<j} \beta_{i j}\left|b_{i j}\right|^{2}+\sum_{i} \lambda_{i} b_{i i}^{2} .
\end{aligned}
$$

Proof. It is plain to see

$$
\operatorname{Tr}\left(\rho^{\alpha} A \rho^{1-\alpha} B\right)=\sum_{i, j=1}^{n} \lambda_{i}^{\alpha} \lambda_{j}^{1-\alpha} a_{i j} \overline{b_{i j}}=\sum_{i \neq j} \lambda_{i}^{\alpha} \lambda_{j}^{1-\alpha} a_{i j} \overline{b_{i j}}+\sum_{i} \lambda_{i} a_{i i} b_{i i}
$$

because diagonal components $a_{i i}, b_{i i}$ are real. Then, the trivial fact $\Re\left(a_{j i} \overline{b_{j i}}\right)=$ $\Re\left(\overline{a_{i j} \overline{b_{i j}}}\right)=\Re\left(a_{i j} \overline{b_{i j}}\right)$ yields

$$
\begin{aligned}
& \Re \operatorname{Tr}\left(\rho^{\alpha} A \rho^{1-\alpha} B\right)=\sum_{i \neq j} \lambda_{i}^{\alpha} \lambda_{j}^{1-\alpha} \Re\left(a_{i j} \overline{b_{i j}}\right)+\sum_{i} \lambda_{i} a_{i i} b_{i i} \\
& =\sum_{i<j}\left(\lambda_{i}^{\alpha} \lambda_{j}^{1-\alpha}+\lambda_{j}^{\alpha} \lambda_{i}^{1-\alpha}\right) \Re\left(a_{i j} \overline{b_{i j}}\right)+\sum_{i} \lambda_{i} a_{i i} b_{i i},
\end{aligned}
$$

which is exactly the second equation in the lemma. The first is just a special case of the second (with $\alpha=1$ ).

It is easy to see

$$
\left\{\begin{array}{l}
\operatorname{Corr}_{\rho}(A+a 1, B+b 1 ; \alpha)=\operatorname{Corr}_{\rho}(A, B ; \alpha) \\
\operatorname{Var}_{\rho}(A+a 1, B+b 1)=\operatorname{Var}_{\rho}(A, B)
\end{array}\right.
$$

for $a, b \in \mathbf{R}$. Thanks to this invariance, to prove (4) or its one-parameter version, (by considering $A-\operatorname{Tr}(\rho A) 1$ and $B-\operatorname{Tr}(\rho B) 1)$ we may and do assume $\operatorname{Tr}(\rho A)=$ $\operatorname{Tr}(\rho B)=0$. This assumption means

$$
\operatorname{Var}_{\rho} A=\operatorname{Tr}\left(\rho A^{2}\right), \operatorname{Var}_{\rho} B=\operatorname{Tr}\left(\rho B^{2}\right), \operatorname{Cov}_{\rho}(A, B)=\operatorname{Tr}(\rho A B)
$$

and hence

$$
\begin{gathered}
\operatorname{Var}_{\rho} A \cdot \operatorname{Var}_{\rho} B-\frac{1}{4}\left|\operatorname{Cov}_{\rho}(A, B)+\operatorname{Cov}_{\rho}(B, A)\right|^{2} \\
=\operatorname{Tr}\left(\rho A^{2}\right) \cdot \operatorname{Tr}\left(\rho B^{2}\right)-(\Re \operatorname{Tr}(\rho A B))^{2}
\end{gathered}
$$


On the other hand, we compute

$$
\begin{gathered}
\mathrm{I}(\rho, A ; \alpha) \cdot \mathrm{I}(\rho, B ; \alpha)-\frac{1}{4}\left|\operatorname{Corr}_{\rho}(A, B ; \alpha)+\operatorname{Corr}_{\rho}(B, A ; \alpha)\right|^{2} \\
=\left(\operatorname{Tr}\left(\rho A^{2}\right)-\operatorname{Tr}\left(\rho^{\alpha} A \rho^{1-\alpha} A\right)\left(\operatorname{Tr}\left(\rho B^{2}\right)-\operatorname{Tr}\left(\rho^{\alpha} B \rho^{1-\alpha} B\right)\right.\right. \\
-\left(\Re \operatorname{Tr}(\rho A B)-\Re \operatorname{Tr}\left(\rho^{\alpha} A \rho^{1-\alpha} B\right)\right)^{2} \\
=\operatorname{Tr}\left(\rho A^{2}\right) \cdot \operatorname{Tr}\left(\rho B^{2}\right)+\operatorname{Tr}\left(\rho^{\alpha} A \rho^{1-\alpha} A\right) \cdot \operatorname{Tr}\left(\rho^{\alpha} B \rho^{1-\alpha} B\right) \\
-\operatorname{Tr}\left(\rho A^{2}\right) \cdot \operatorname{Tr}\left(\rho^{\alpha} B \rho^{1-\alpha} B\right)-\operatorname{Tr}\left(\rho B^{2}\right) \cdot \operatorname{Tr}\left(\rho^{\alpha} A \rho^{1-\alpha} A\right) \\
-(\Re \operatorname{Tr}(\rho A B))^{2}-\left(\Re \operatorname{Tr}\left(\rho^{\alpha} A \rho^{1-\alpha} B\right)\right)^{2} \\
+2(\Re \operatorname{Tr}(\rho A B))\left(\Re \operatorname{Tr}\left(\rho^{\alpha} A \rho^{1-\alpha} B\right)\right) .
\end{gathered}
$$

We set

$$
\begin{aligned}
& I=\operatorname{Tr}\left(\rho A^{2}\right) \cdot \operatorname{Tr}\left(\rho^{\alpha} B \rho^{1-\alpha} B\right)+\operatorname{Tr}\left(\rho B^{2}\right) \cdot \operatorname{Tr}\left(\rho^{\alpha} A \rho^{1-\alpha} A\right), \\
& J=2\left(\Re \operatorname{Tr}\left(\rho^{\alpha} A \rho^{1-\alpha} B\right)\right)(\Re \operatorname{Tr}(\rho A B)), \\
& K=\operatorname{Tr}\left(\rho^{\alpha} A \rho^{1-\alpha} A\right) \cdot \operatorname{Tr}\left(\rho^{\alpha} B \rho^{1-\alpha} B\right)-\left(\Re \operatorname{Tr}\left(\rho^{\alpha} A \rho^{1-\alpha} B\right)\right)^{2},
\end{aligned}
$$

and observe that (4) (or rather its one-parameter version) means $I-J-K \geq 0$.

Note that $I$ consists of products of "first terms" (i.e., off-diagonal entries), products of "second terms" (i.e., diagonal entries), and "cross terms". More explicitly, thanks to Lemma 3 we have

$$
I=I_{11}+I_{12}+I_{22}
$$

with

$$
\begin{aligned}
I_{11}= & \left(\sum_{i<j} \alpha_{i j}\left|a_{i j}\right|^{2}\right)\left(\sum_{i<j} \beta_{i j}\left|b_{i j}\right|^{2}\right)+\left(\sum_{i<j} \alpha_{i j}\left|b_{i j}\right|^{2}\right)\left(\sum_{i<j} \beta_{i j}\left|a_{i j}\right|^{2}\right), \\
I_{12}= & \left(\sum_{i<j} \alpha_{i j}\left|a_{i j}\right|^{2}\right)\left(\sum_{i} \lambda_{i} b_{i i}^{2}\right)+\left(\sum_{i<j} \beta_{i j}\left|b_{i j}\right|^{2}\right)\left(\sum_{i} \lambda_{i} a_{i i}^{2}\right) \\
& +\left(\sum_{i<j} \alpha_{i j}\left|b_{i j}\right|^{2}\right)\left(\sum_{i} \lambda_{i} a_{i i}^{2}\right)+\left(\sum_{i<j} \beta_{i j}\left|a_{i j}\right|^{2}\right)\left(\sum_{i} \lambda_{i} b_{i i}^{2}\right) \\
= & \left(\sum_{i<j}\left(\alpha_{i j}+\beta_{i j}\right)\left|a_{i j}\right|^{2}\right)\left(\sum_{i} \lambda_{i} b_{i i}^{2}\right)+\left(\sum_{i<j}\left(\alpha_{i j}+\beta_{i j}\right)\left|b_{i j}\right|^{2}\right)\left(\sum_{i} \lambda_{i} a_{i i}^{2}\right), \\
I_{22}= & 2\left(\sum_{i} \lambda_{i} a_{i i}^{2}\right)\left(\sum_{i} \lambda_{i} b_{i i}^{2}\right) .
\end{aligned}
$$

It is plain to see

$$
I_{11}=\sum_{i<j, k<\ell}\left(\alpha_{i j} \beta_{k \ell}+\alpha_{k \ell} \beta_{i j}\right)\left|a_{i j}\right|^{2}\left|b_{k \ell}\right|^{2}
$$


The coefficients here are unchanged under the transpositions $(i, j) \leftrightarrow(k, \ell)$, which enables us to rewrite $I_{11}$ in the following "more symmetric" fashion:

$$
I_{11}=\frac{1}{2} \sum_{i<j, k<\ell}\left(\alpha_{i j} \beta_{k \ell}+\alpha_{k \ell} \beta_{i j}\right)\left(\left|a_{i j}\right|^{2}\left|b_{k \ell}\right|^{2}+\left|a_{k \ell}\right|^{2}\left|b_{i j}\right|^{2}\right) \text {. }
$$

Similarly, we express $J, K$ as

$$
J=J_{11}+J_{12}+J_{22}, \quad K=K_{11}+K_{12}+K_{22}
$$

and (to prove $I-J-K \geq 0$ ) we will look at

$$
I_{11}-J_{11}-K_{11}, I_{12}-J_{12}-K_{12}, I_{22}-J_{22}-K_{22}
$$

separately. Let us recall

$$
J=2\left(\sum_{i<j} \beta_{i j} \Re\left(a_{i j} \overline{b_{i j}}\right)+\sum_{i} \lambda_{i} a_{i i} b_{i i}\right)\left(\sum_{i<j} \alpha_{i j} \Re\left(a_{i j} \overline{b_{i j}}\right)+\sum_{i} \lambda_{i} a_{i i} b_{i i}\right)
$$

(see Lemma 3). From this expression we conclude

$$
\begin{aligned}
J_{11} & =2\left(\sum_{i<j} \beta_{i j} \Re\left(a_{i j} \overline{b_{i j}}\right)\right)\left(\sum_{i<j} \alpha_{i j} \Re\left(a_{i j} \overline{b_{i j}}\right)\right) \\
J_{12} & =2\left(\sum_{i<j} \beta_{i j} \Re\left(a_{i j} \overline{b_{i j}}\right)\right)\left(\sum_{i} \lambda_{i} a_{i i} b_{i i}\right)+2\left(\sum_{i<j} \alpha_{i j} \Re\left(a_{i j} \overline{b_{i j}}\right)\right)\left(\sum_{i} \lambda_{i} a_{i i} b_{i i}\right) \\
& =2\left(\sum_{i<j}\left(\alpha_{i j}+\beta_{i j}\right) \Re\left(a_{i j} \overline{b_{i j}}\right)\right)\left(\sum_{i} \lambda_{i} a_{i i} b_{i i}\right), \\
J_{22} & =2\left(\sum_{i} \lambda_{i} a_{i i} b_{i i}\right)^{2} .
\end{aligned}
$$

We have

$$
\begin{aligned}
J_{11} & =2 \sum_{i<j, k<\ell} \alpha_{i j} \beta_{k \ell} \Re\left(a_{i j} \overline{b_{i j}}\right) \Re\left(a_{k \ell} \overline{b_{k \ell}}\right) \\
& =\sum_{i<j, k<\ell}\left(\alpha_{i j} \beta_{k \ell}+\alpha_{k \ell} \beta_{i j}\right) \Re\left(a_{i j} \overline{b_{i j}}\right) \Re\left(a_{k \ell} \overline{b_{k \ell}}\right)
\end{aligned}
$$

because the products $\Re\left(a_{i j} \overline{b_{i j}}\right) \Re\left(a_{k \ell} \overline{b_{k \ell}}\right)$ are unchanged under the transpositions $(i, j) \leftrightarrow(k, \ell)$ 
It remains to compute $K_{11}, K_{12}, K_{22}$. Lemma 3 implies

$$
\begin{aligned}
K=\left(\sum_{i<j} \beta_{i j}\left|a_{i j}\right|^{2}\right. & \left.+\sum_{i} \lambda_{i} a_{i i}^{2}\right)\left(\sum_{i<j} \beta_{i j}\left|b_{i j}\right|^{2}+\sum_{i} \lambda_{i} b_{i i}^{2}\right) \\
& -\left(\sum_{i<j} \beta_{i j} \Re\left(a_{i j} \overline{b_{i j}}\right)+\sum_{i} \lambda_{i} a_{i i} b_{i i}\right)^{2}
\end{aligned}
$$

and we observe

$$
\begin{aligned}
& K_{11}=\left(\sum_{i<j} \beta_{i j}\left|a_{i j}\right|^{2}\right)\left(\sum_{i<j} \beta_{i j}\left|b_{i j}\right|^{2}\right)-\left(\sum_{i<j} \beta_{i j} \Re\left(a_{i j} \overline{b_{i j}}\right)\right)^{2}, \\
& K_{12}=\left(\sum_{i<j} \beta_{i j}\left|a_{i j}\right|^{2}\right)\left(\sum_{i} \lambda_{i} b_{i i}^{2}\right)+\left(\sum_{i<j} \beta_{i j}\left|b_{i j}\right|^{2}\right)\left(\sum_{i} \lambda_{i} a_{i i}^{2}\right) \\
&-2\left(\sum_{i<j} \beta_{i j} \Re\left(a_{i j} \overline{b_{i j}}\right)\right)\left(\sum_{i} \lambda_{i} a_{i i} b_{i i}\right), \\
& K_{22}=\left(\sum_{i} \lambda_{i} a_{i i}^{2}\right)\left(\sum_{i} \lambda_{i} b_{i i}^{2}\right)-\left(\sum_{i} \lambda_{i} a_{i i} b_{i i}\right)^{2} .
\end{aligned}
$$

We note

$$
\begin{aligned}
K_{11}= & \sum_{i<j, k<\ell} \beta_{i j} \beta_{k \ell}\left|a_{i j}\right|^{2}\left|b_{k \ell}\right|^{2}-\sum_{i<j, k<\ell} \beta_{i j} \beta_{k \ell} \Re\left(a_{i j} \overline{b_{i j}}\right) \Re\left(a_{k \ell} \overline{b_{k \ell}}\right) \\
= & \frac{1}{2} \sum_{i<j, k<\ell} \beta_{i j} \beta_{k \ell}\left(\left|a_{i j}\right|^{2}\left|b_{k \ell}\right|^{2}+\left|a_{k \ell}\right|^{2}\left|b_{i j}\right|^{2}\right) \\
& \quad-\sum_{i<j, k<\ell} \beta_{i j} \beta_{k \ell} \Re\left(a_{i j} \overline{b_{i j}}\right) \Re\left(a_{k \ell} \overline{b_{k \ell}}\right) \\
= & \frac{1}{2} \sum_{i<j, k<\ell} \beta_{i j} \beta_{k \ell}\left(\left|a_{i j}\right|^{2}\left|b_{k \ell}\right|^{2}+\left|a_{k \ell}\right|^{2}\left|b_{i j}\right|^{2}-2 \Re\left(a_{i j} \overline{b_{i j}}\right) \Re\left(a_{k \ell} \overline{b_{k \ell}}\right)\right),
\end{aligned}
$$

where the second equality follows from the invariance of the products $\beta_{i j} \beta_{k \ell}$ under the transpositions $(i, j) \leftrightarrow(k, \ell)$.

We are now ready to look at $I_{i j}-J_{i j}-K_{i j}((i, j)=(1,1),(1,2),(22))$. The easiest one to deal with is $I_{22}-J_{22}-K_{22}$. Indeed, it is plain to see

$$
I_{22}-J_{22}-K_{22}=\left(\sum_{i} \lambda_{i} a_{i i}^{2}\right)\left(\sum_{i} \lambda_{i} b_{i i}^{2}\right)-\left(\sum_{i} \lambda_{i} a_{i i} b_{i i}\right)^{2} .
$$

From (6) and (7) we get

$$
I_{11}-J_{11}=\frac{1}{2} \sum_{i<j, k<\ell}\left(\alpha_{i j} \beta_{k \ell}+\alpha_{k \ell} \beta_{i j}\right)\left(\left|a_{i j}\right|^{2}\left|b_{k \ell}\right|^{2}+\left|a_{k \ell}\right|^{2}\left|b_{i j}\right|^{2}-2 \Re\left(a_{i j} \overline{b_{i j}}\right) \Re\left(a_{k \ell} \overline{b_{k \ell}}\right)\right) \text {. }
$$


Therefore, (8) implies

$$
\begin{aligned}
I_{11}-J_{11}-K_{11}= & \frac{1}{2} \sum_{i<j, k<\ell}\left(\beta_{i j}\left(\alpha_{k \ell}-\frac{1}{2} \beta_{k \ell}\right)+\beta_{k \ell}\left(\alpha_{i j}-\frac{1}{2} \beta_{i j}\right)\right) \\
& \quad \times\left(\left|a_{i j}\right|^{2}\left|b_{k \ell}\right|^{2}+\left|a_{k \ell}\right|^{2}\left|b_{i j}\right|^{2}-2 \Re\left(a_{i j} \overline{b_{i j}}\right) \Re\left(a_{k \ell} \overline{b_{k \ell}}\right)\right) .
\end{aligned}
$$

Finally, all the cross terms sum up to

$$
\begin{aligned}
& I_{12}- J_{12}-K_{12} \\
&=\left(\sum_{i<j}\left(\alpha_{i j}+\beta_{i j}\right)\left|a_{i j}\right|^{2}\right)\left(\sum_{i} \lambda_{i} b_{i i}^{2}\right)+\left(\sum_{i<j}\left(\alpha_{i j}+\beta_{i j}\right)\left|b_{i j}\right|^{2}\right)\left(\sum_{i} \lambda_{i} a_{i i}^{2}\right) \\
&-2\left(\sum_{i<j}\left(\alpha_{i j}+\beta_{i j}\right) \Re\left(a_{i j} \overline{b_{i j}}\right)\right)\left(\sum_{i} \lambda_{i} a_{i i} b_{i i}\right) \\
&-\left(\sum_{i<j} \beta_{i j}\left|a_{i j}\right|^{2}\right)\left(\sum_{i} \lambda_{i} b_{i i}^{2}\right)-\left(\sum_{i<j} \beta_{i j}\left|b_{i j}\right|^{2}\right)\left(\sum_{i} \lambda_{i} a_{i i}^{2}\right) \\
&=\left.\left(\sum_{i<j} \alpha_{i j}\left|a_{i j}\right|^{2}\right)\left(\sum_{i} \lambda_{i} b_{i i}^{2}\right)+\left(\sum_{i j} \overline{b_{i j}}\right)\right)\left(\sum_{i} \lambda_{i j}\left|b_{i j}\right|^{2}\right)\left(\sum_{i} b_{i i} \lambda_{i} a_{i i}^{2}\right) \\
&-2\left(\sum_{i<j} \alpha_{i j} \Re\left(a_{i j} \overline{b_{i j}}\right)\right)\left(\sum_{i} \lambda_{i} a_{i i} b_{i i}\right) .
\end{aligned}
$$

The first term in the above last expression can be rewritten as

$$
\left(\sum_{i<j} \alpha_{i j}\left|a_{i j}\right|^{2}\right)\left(\sum_{i} \lambda_{i} b_{i i}^{2}\right)=\sum_{k} \lambda_{k}\left(\sum_{i<j} \alpha_{i j} b_{k k}^{2}\left|a_{i j}\right|^{2}\right) .
$$

Note that the first factor in each of the last three terms contains the identical coefficients $\alpha_{i j}$, which enables us to rearrange the above sum into

$$
\begin{aligned}
I_{12}-J_{12}-K_{12} & =\sum_{k} \lambda_{k}\left(\sum_{i<j} \alpha_{i j}\left(b_{k k}^{2}\left|a_{i j}\right|^{2}+a_{k k}^{2}\left|b_{i j}\right|^{2}-2 a_{k k} b_{k k} \Re\left(a_{i j} \overline{b_{i j}}\right)\right)\right) \\
& =\sum_{k} \lambda_{k}\left(\sum_{i<j} \alpha_{i j}\left|b_{k k} a_{i j}-a_{k k} b_{i j}\right|^{2}\right) .
\end{aligned}
$$

Summing up the computations so far (see (9), (10) and (11)) and recalling the definitions of $\alpha_{i j}, \beta_{i j}$ in Lemma 3, we conclude 
Lemma 4. We have

$$
\begin{aligned}
& I-J-K \\
& =\frac{1}{2} \sum_{i<j, k<\ell}\left\{\left(\lambda_{i}^{\alpha} \lambda_{j}^{1-\alpha}+\lambda_{i}^{1-\alpha} \lambda_{j}^{\alpha}\right)\left(\lambda_{k}+\lambda_{\ell}-\frac{1}{2}\left(\lambda_{k}^{\alpha} \lambda_{\ell}^{1-\alpha}+\lambda_{k}^{1-\alpha} \lambda_{\ell}^{\alpha}\right)\right)\right. \\
& \left.+\left(\lambda_{k}^{\alpha} \lambda_{\ell}^{1-\alpha}+\lambda_{k}^{1-\alpha} \lambda_{\ell}^{\alpha}\right)\left(\lambda_{i}+\lambda_{j}-\frac{1}{2}\left(\lambda_{i}^{\alpha} \lambda_{j}^{1-\alpha}+\lambda_{i}^{1-\alpha} \lambda_{j}^{\alpha}\right)\right)\right\} \\
& \times\left(\left|a_{i j}\right|^{2}\left|b_{k \ell}\right|^{2}+\left|a_{k \ell}\right|^{2}\left|b_{i j}\right|^{2}-2 \Re\left(a_{i j} \overline{b_{i j}}\right) \Re\left(a_{k \ell} \overline{b_{k \ell}}\right)\right) \\
& +\sum_{k} \lambda_{k}\left(\sum_{i<j}\left(\lambda_{i}+\lambda_{j}\right)\left|b_{k k} a_{i j}-a_{k k} b_{i j}\right|^{2}\right) \\
& +\left(\sum_{i} \lambda_{i} a_{i i}^{2}\right)\left(\sum_{i} \lambda_{i} b_{i i}^{2}\right)-\left(\sum_{i} \lambda_{i} a_{i i} b_{i i}\right)^{2} .
\end{aligned}
$$

The last part $\left(\sum_{i} \lambda_{i} a_{i i}^{2}\right)\left(\sum_{i} \lambda_{i} b_{i i}^{2}\right)-\left(\sum_{i} \lambda_{i} a_{i i} b_{i i}\right)^{2}$ is non-negative by the CauchySchwarz inequality while the arithmetic-geometric mean inequality guarantees

$$
\begin{aligned}
\frac{\left|a_{i j}\right|^{2}\left|b_{k \ell}\right|^{2}+\left|a_{k \ell}\right|^{2}\left|b_{i j}\right|^{2}}{2} & \geq\left|a_{i j}\right|\left|b_{k \ell}\right| \times\left|a_{k \ell}\right|\left|b_{i j}\right|=\left|a_{i j} \overline{b_{i j}}\right| \times\left|a_{k \ell} \overline{b_{k \ell}}\right| \\
& \geq\left|\Re\left(a_{i j} \overline{b_{i j}}\right)\right| \times\left|\Re\left(a_{k \ell} \overline{b_{k \ell}}\right)\right| \\
& \geq \Re\left(a_{i j} \overline{b_{i j}}\right) \Re\left(a_{k \ell} \overline{b_{k \ell}}\right) .
\end{aligned}
$$

It remains to check behavior of the coefficient

$$
\begin{array}{r}
\tilde{f}(\alpha)=\left(\lambda_{i}^{\alpha} \lambda_{j}^{1-\alpha}+\lambda_{i}^{1-\alpha} \lambda_{j}^{\alpha}\right)\left(\lambda_{k}+\lambda_{\ell}-\frac{1}{2}\left(\lambda_{k}^{\alpha} \lambda_{\ell}^{1-\alpha}+\lambda_{k}^{1-\alpha} \lambda_{\ell}^{\alpha}\right)\right) \\
+\left(\lambda_{k}^{\alpha} \lambda_{\ell}^{1-\alpha}+\lambda_{k}^{1-\alpha} \lambda_{\ell}^{\alpha}\right)\left(\lambda_{i}+\lambda_{j}-\frac{1}{2}\left(\lambda_{i}^{\alpha} \lambda_{j}^{1-\alpha}+\lambda_{i}^{1-\alpha} \lambda_{j}^{\alpha}\right)\right)
\end{array}
$$

(for each fixed $i, j, k, \ell$ ) appearing in the first sum. We obviously have the symmetry $\tilde{f}(\alpha)=\tilde{f}(1-\alpha)(\alpha \in(0,1))$, and for the special value $\alpha=1 / 2$ we have

$$
\tilde{f}(1 / 2)=2 \sqrt{\lambda_{i} \lambda_{j}}\left(\lambda_{k}+\lambda_{\ell}-\sqrt{\lambda_{k} \lambda_{\ell}}\right)+2 \sqrt{\lambda_{k} \lambda_{\ell}}\left(\lambda_{i}+\lambda_{j}-\sqrt{\lambda_{i} \lambda_{j}}\right)>0,
$$

showing $I-J-K \geq 0$ for $\alpha=1 / 2$, i.e., the conjectured inequality (4) is valid. With $x=\lambda_{j} / \lambda_{i}$ and $y=\lambda_{\ell} / \lambda_{k}$ we have

$$
\begin{aligned}
\frac{\tilde{f}(\alpha)}{\lambda_{i} \lambda_{k}}= & \left(x^{1-\alpha}+x^{\alpha}\right)\left(1+y-\frac{1}{2}\left(y^{1-\alpha}+y^{\alpha}\right)\right) \\
& +\left(y^{1-\alpha}+y^{\alpha}\right)\left(1+x-\frac{1}{2}\left(x^{1-\alpha}+x^{\alpha}\right)\right) \\
= & \sqrt{x y}\left(\left(x^{1 / 2-\alpha}+x^{\alpha-1 / 2}\right)\left(y^{1 / 2}+y^{-1 / 2}-\frac{1}{2}\left(y^{1 / 2-\alpha}+y^{\alpha-1 / 2}\right)\right)\right. \\
& \left.+\left(y^{1 / 2-\alpha}+y^{\alpha-1 / 2}\right)\left(x^{1 / 2}+x^{-1 / 2}-\frac{1}{2}\left(x^{1 / 2-\alpha}+x^{\alpha-1 / 2}\right)\right)\right) .
\end{aligned}
$$


Therefore, (with $s=\frac{1}{2} \log x$ and $t=\frac{1}{2} \log y$ ) we need to consider the function

$$
\begin{aligned}
f(\alpha)=\cosh & ((2 \alpha-1) s)(2 \cosh (t)-\cosh ((2 \alpha-1) t)) \\
& +\cosh ((2 \alpha-1) t)(2 \cosh (s)-\cosh ((2 \alpha-1) s)) .
\end{aligned}
$$

It is elementary to see

$$
\begin{aligned}
f^{\prime}(\alpha)=4 s \sinh ((2 \alpha-1) s)(\cosh (t)-\cosh ((2 \alpha-1) t)) \\
+4 t \sinh ((2 \alpha-1) t)(\cosh (s)-\cosh ((2 \alpha-1) s)),
\end{aligned}
$$

and we note

$$
\left\{\begin{array}{l}
\cosh (t) \geq \cosh ((2 \alpha-1) t) \text { and } \cosh (s) \geq \cosh ((2 \alpha-1) s) \text { for } \alpha \in(0,1) \\
s \sinh ((2 \alpha-1) s) \geq 0 \text { and } t \sinh ((2 \alpha-1) t) \geq 0 \text { for } \alpha \in[1 / 2,1)
\end{array}\right.
$$

showing that $f(\alpha)$ (and hence $\tilde{f}(\alpha)$ as well) is increasing on the interval $[1 / 2,1)$.

From Lemma 4 and the discussions so far we obtain the following main result in the article:

Theorem 5. We assume that $A, B$ are self-adjoint matrices and $\rho$ is a density matrix. Then, the quantity

$$
\begin{aligned}
F(\alpha)=\left(\operatorname{Var}_{\rho} A \cdot \operatorname{Var}_{\rho} B-\frac{1}{4}\left|\operatorname{Cov}_{\rho}(A, B)+\operatorname{Cov}_{\rho}(B, A)\right|^{2}\right) \\
\\
\quad-\left(I(\rho, A ; \alpha) \cdot I(\rho, B ; \alpha)-\frac{1}{4}\left|\operatorname{Corr}_{\rho}(A, B ; \alpha)+\operatorname{Corr}_{\rho}(B, A ; \alpha)\right|^{2}\right)
\end{aligned}
$$

defined for $\alpha \in(0,1)$ satisfies $F(1 / 2) \geq 0$, that is, the conjectured inequality (4) holds true. Moreover, the function $F(\alpha)(=F(1-\alpha))$ is monotone increasing on the right half interval $[1 / 2,1)$.

The equality condition for the inequality in the theorem can be also determined (under the additional assumption that $\rho$ is invertible) from the explicit expression given by Lemma 4.

Proposition 6. We assume that the density matrix $\rho$ is invertible and $\alpha \in(0,1)$. Then, the inequality in the preceding theorem reduces to the equality

$$
\begin{aligned}
& \operatorname{Var}_{\rho} A \cdot \operatorname{Var}_{\rho} B-\frac{1}{4}\left|\operatorname{Cov}_{\rho}(A, B)+\operatorname{Cov}_{\rho}(B, A)\right|^{2} \\
& \quad=I(\rho, A ; \alpha) \cdot I(\rho, B ; \alpha)-\frac{1}{4}\left|\operatorname{Corr}_{\rho}(A, B ; \alpha)+\operatorname{Corr}_{\rho}(B, A ; \alpha)\right|^{2}
\end{aligned}
$$

for $A, B \in M_{n}(\mathbf{C})_{s a}$ if and only if the two matrices $A-\operatorname{Tr}(\rho A) 1$ and $B-\operatorname{Tr}(\rho B) 1$ are proportional.

Proof. Thanks to the invariance (5) we may and do assume $\operatorname{Tr}(\rho A)=\operatorname{Tr}(\rho B)=0$ in what follows. When $A, B$ are proportional, the expression in Lemma 4 readily shows the equality in question. We conversely assume this equality in the rest (and will show that $A, B$ are proportional), which forces that all the three parts of the expression in Lemma 4 must be zero. 
Case 1. $\left(a_{11}, a_{22}, \cdots, a_{n n}\right)=0$ (as a vector) and $\left(b_{11}, b_{22}, \cdots, b_{n n}\right) \neq 0$ The second part $\sum_{k} \lambda_{k}\left(\sum_{i<j}\left(\lambda_{i}+\lambda_{j}\right)\left|b_{k k} a_{i j}-a_{k k} b_{i j}\right|^{2}\right)$ of the expression in Lemma 4 must be zero. Thus, by choosing $k \in\{1,2, \cdots, n\}$ with $b_{k k} \neq 0$ we must have $\sum_{i<j}\left(\lambda_{i}+\lambda_{j}\right) b_{k k}^{2}\left|a_{i j}\right|^{2}=0$. Thus, we get $a_{i j}=0$ for each $i<j$, showing $A=0$.

Case 2. $\left(b_{11}, b_{22}, \cdots, b_{n n}\right)=0$ and $\left(a_{11}, a_{22}, \cdots, a_{n n}\right) \neq 0$

By exchanging the roles of $a$ 's and $b$ 's in the previous case, we get $B=0$.

Case 3. $\left(a_{11}, a_{22}, \cdots, a_{n n}\right) \neq 0$ and $\left(b_{11}, b_{22}, \cdots, b_{n n}\right) \neq 0$

The last part $\left(\sum_{i} \lambda_{i} a_{i i}^{2}\right)\left(\sum_{i} \lambda_{i} b_{i i}^{2}\right)-\left(\sum_{i} \lambda_{i} a_{i i} b_{i i}\right)^{2}$ of the expression in Lemma 4 is zero so that we must have $a_{k k}=\gamma b_{k k}(k=1,2, \cdots, n)$ for some $\gamma \in \mathbf{R} \backslash\{0\}$. By choosing $b_{k k} \neq 0$, from the second part we get

$$
0=\sum_{i<j}\left(\lambda_{i}+\lambda_{j}\right)\left|b_{k k} a_{i j}-a_{k k} b_{i j}\right|^{2}=\sum_{i<j}\left(\lambda_{i}+\lambda_{j}\right) b_{k k}^{2}\left|a_{i j}-\gamma b_{i j}\right|^{2},
$$

showing $a_{i j}=\gamma b_{i j}$ for each $i<j$ and hence $A=\gamma B$.

Case 4. $\left(a_{11}, a_{22}, \cdots, a_{n n}\right)=\left(b_{11}, b_{22}, \cdots, b_{n n}\right)=0$

We can assume $A \neq 0$ and $B \neq 0$ since we have nothing to prove otherwise. Only the first part of the expression in Lemma 4 provides us meaning information in the present case. Namely, all the three inequalities in (12) have to be equalities (for each $i<j$ and $k<\ell$ ). Let us assume $a_{i j} \neq 0$ for some $i<j$. The equality condition for the first inequality in (12) (i.e., the arithmetic-geometric mean inequality) says

$$
\left|a_{i j} b_{k \ell}\right|=\left|a_{k \ell} b_{i j}\right| \quad(\text { for each } k<\ell) .
$$

By choosing $k<\ell$ with $b_{k \ell} \neq 0$, we observe $b_{i j} \neq 0$ (and $a_{k \ell} \neq 0$ ). By the symmetric reasoning, $b_{i j} \neq 0$ also implies $a_{i j} \neq 0$. For convenience we set

$$
\Lambda=\left\{(i, j) ; 1 \leq i<j \leq n \text { and } a_{i j} \neq 0\right\}\left(=\left\{(i, j) ; 1 \leq i<j \leq n \text { and } b_{i j} \neq 0\right\}\right) .
$$

The above equality condition (13) also yields that ratios $\left|a_{i j}\right| /\left|b_{i j}\right|$ are constant, i.e., $\left|a_{i j}\right|=\gamma\left|b_{i j}\right|$ (for each $(i, j) \in \Lambda$ ) for some $\gamma>0$. It is elementary to see

$$
\left|z_{1} \overline{z_{2}}\right|=\left|\Re\left(z_{1} \overline{z_{2}}\right)\right| \text { if and only if } \arg z_{1}=\arg z_{2} \text { or } \arg z_{1}=\arg z_{2}+\pi \text {. }
$$

Thus, the requirement that the second inequality in (12) must be the equality further forces $a_{i j}= \pm \gamma b_{i j}$ (for each $(i, j) \in \Lambda$ ). The parity here depends upon $(i, j) \in \Lambda$ at this stage, but $\gamma$ and $-\gamma$ cannot actually mix because the third inequality in (12) is also the equality. Indeed, if both of $a_{i j}=\gamma b_{i j}$ and $a_{k \ell}=-\gamma b_{k \ell}$ occured for some $(i, j),(k, \ell) \in \Lambda$, then we would get two different values

$$
\begin{aligned}
& \Re\left(a_{i j} \overline{b_{i j}}\right) \Re\left(a_{k \ell} \overline{b_{k \ell}}\right)=\left(\gamma\left|b_{i j}\right|^{2}\right)\left(-\gamma\left|b_{k \ell}\right|^{2}\right)=-\gamma^{2}\left|b_{i j}\right|^{2}\left|b_{k \ell}\right|^{2}, \\
& \left|\Re\left(a_{i j} \overline{b_{i j}}\right)\right| \times\left|\Re\left(a_{k \ell} \overline{b_{k \ell}}\right)\right|=\gamma^{2}\left|b_{i j}\right|^{2}\left|b_{k \ell}\right|^{2},
\end{aligned}
$$

a contradiction. Thus, we conclude either $A=\gamma B$ or $A=-\gamma B$, and we are done. 
Each of the quantities appearing in the theorem and the proposition is known to make a perfect sense in the general von Neumann algebra setting, and the notion of skew information was indeed useful in [3] (where the homogeneity of the state space of a type $I I I_{1}$ factor was established). Our arguments presented in this section depend heavily upon manipulations of matrix components so that our method cannot be employed in the general setting. A suitable way to avoid this difficulty has to be found, and the author hopes to be able to come back to this problem.

\section{Discussions}

\section{1.}

In $[5$, Theorem 2 in p.1671] the inequality

$$
\frac{1}{4}|\operatorname{Tr}(\rho[A, B])|^{2}+\frac{1}{16}|\mathrm{I}(\rho, A+B)-\mathrm{I}(\rho, A-B)|^{2} \leq \mathrm{I}(\rho, A) \cdot \mathrm{I}(\rho, B)
$$

was claimed. The real and imaginary parts of $\operatorname{Corr}_{\rho}(A, B)$ are given by

$$
\left\{\begin{array}{l}
\frac{1}{2}\left(\operatorname{Corr}_{\rho}(A, B)+\operatorname{Corr}_{\rho}(B, A)\right)=\frac{1}{4}(\mathrm{I}(\rho, A+B)-\mathrm{I}(\rho, A-B)), \\
\frac{1}{2 i}\left(\operatorname{Corr}_{\rho}(A, B)-\operatorname{Corr}_{\rho}(B, A)\right)=\frac{1}{2 i} \operatorname{Tr}(\rho[A, B]),
\end{array}\right.
$$

and (14) is actually equivalent to the following Cauchy-Schwarz tye estimate:

$$
\left|\operatorname{Corr}_{\rho}(A, B)\right|^{2} \leq \operatorname{Corr}_{\rho}(A, A) \cdot \operatorname{Corr}_{\rho}(B, B)(=\mathrm{I}(\rho A) \cdot \mathrm{I}(\rho, B)) .
$$

However, the presented argument in [5] has a gap (see the paragraph right before Remark 2), and (14) (i.e., the above Cauchy-Schwarz type estimate) is false even for $2 \times 2$ matrices.

To see this, let us set

$$
\rho=\left[\begin{array}{cc}
\lambda_{1} & 0 \\
0 & \lambda_{2}
\end{array}\right], \quad A=\left[\begin{array}{cc}
a_{11} & a \\
\bar{a} & a_{22}
\end{array}\right], \quad B=\left[\begin{array}{cc}
b_{11} & b \\
\bar{b} & b_{22}
\end{array}\right]
$$

with $a_{i i}, b_{i i} \in \mathbf{R}$ and $\lambda_{i}>0, \lambda_{1}+\lambda_{2}=1$. Lemma 3 (or direct calculations) shows

$$
\begin{aligned}
& \operatorname{Tr}(\rho A B)=\lambda_{1} a_{11} b_{11}+\lambda_{1} a \bar{b}+\lambda_{2} \bar{a} b+\lambda_{2} a_{22} b_{22}, \\
& \operatorname{Tr}\left(\rho^{\alpha} A \rho^{1-\alpha} B\right)=\lambda_{1} a_{11} b_{11}+\lambda_{1}^{\alpha} \lambda_{2}^{1-\alpha} a \bar{b}+\lambda_{1}^{1-\alpha} \lambda_{2}^{\alpha} \bar{a} b+\lambda_{2} a_{22} b_{22} .
\end{aligned}
$$

Since

$$
\begin{aligned}
& \operatorname{Corr}_{\rho}(A, B)=\operatorname{Tr}(\rho A B)-\operatorname{Tr}\left(\rho^{1 / 2} A \rho^{1 / 2} B\right) \\
& \quad=\lambda_{1} a \bar{b}+\lambda_{2} \bar{a} b-\sqrt{\lambda_{1} \lambda_{2}} a \bar{b}-\sqrt{\lambda_{1} \lambda_{2}} \bar{a} b \\
& \quad=\left(\lambda_{1}+\lambda_{2}-2 \sqrt{\lambda_{1} \lambda_{2}}\right) \Re(a \bar{b})+i\left(\lambda_{1}-\lambda_{2}\right) \Im(a \bar{b}),
\end{aligned}
$$


we observe

$$
\begin{aligned}
& \left.\operatorname{Corr}_{\rho}(A, B)\right|^{2}=\left(\lambda_{1}+\lambda_{2}-2 \sqrt{\lambda_{1} \lambda_{2}}\right)^{2}(\Re(a \bar{b}))^{2}+\left(\lambda_{1}-\lambda_{2}\right)^{2}(\Im(a \bar{b}))^{2} \\
& \quad=\left(\sqrt{\lambda_{1}}-\sqrt{\lambda_{2}}\right)^{4}(\Re(a \bar{b}))^{2}+\left(\sqrt{\lambda_{1}}-\sqrt{\lambda_{2}}\right)^{2}\left(\sqrt{\lambda_{1}}+\sqrt{\lambda_{2}}\right)^{2}(\Im(a \bar{b}))^{2}, \\
& \operatorname{Corr}_{\rho}(A, A) \cdot \operatorname{Corr}_{\rho}(B, B)(=\mathrm{I}(\rho, A) \cdot \mathrm{I}(\rho, B)) \\
& \quad=\left.\left(\lambda_{1}+\lambda_{2}-2 \sqrt{\lambda_{1} \lambda_{2}}\right)^{2}|a|^{2}|| b\right|^{2}=\left(\sqrt{\lambda_{1}}-\sqrt{\lambda_{2}}\right)^{4}|a|^{2} \|\left. b\right|^{2} .
\end{aligned}
$$

Consequently, if (14) were valid, then it would mean

$$
\left(\sqrt{\lambda_{1}}-\sqrt{\lambda_{2}}\right)^{2}\left(\sqrt{\lambda_{1}}+\sqrt{\lambda_{2}}\right)^{2}(\Im(a \bar{b}))^{2} \leq\left(\sqrt{\lambda_{1}}-\sqrt{\lambda_{2}}\right)^{4}(\Im(a \bar{b}))^{2} .
$$

But, when $\Im(a \bar{b}) \neq 0$ and $\lambda_{1} \neq \lambda_{2}$, it would be impossible for $\lambda_{1}$ close to $1 / 2$.

\section{2.}

One of the reasons why the inequality in Theorem 5 (with $\alpha=1 / 2$ ) was conjectured in [5] was that this together with (14) would imply Schrödinger's uncertainty principle (explained in Remark 2). However, (14) is not valid, and the best we can hope in this direction is the estimate

$$
\left(\Re \operatorname{Corr}_{\rho}(A, B)\right)^{2} \leq \mathrm{I}(\rho, A) \cdot \mathrm{I}(\rho, B)
$$

(see (2)). Note that the difference between the left side here and that of (14) is

$$
\left(\Im \operatorname{Corr}_{\rho}(A, B)\right)^{2}=(\Im \operatorname{Tr}(\rho A B))^{2}\left(=\frac{1}{4}|\operatorname{Tr}(\rho[A, B])|^{2}\right) .
$$

Therefore, a natural alternative question is whether the estimate

$$
F(1 / 2) \geq\left(\Im \operatorname{Corr}_{\rho}(A, B)\right)^{2}
$$

(which is stronger than that in Theorem 5 by the above difference) is valid.

Actually this estimate is not valid. In fact, for

$$
\rho=\left[\begin{array}{cc}
\lambda_{1} & 0 \\
0 & \lambda_{2}
\end{array}\right], \quad A=\left[\begin{array}{cc}
\lambda_{2} & a \\
\bar{a} & -\lambda_{1}
\end{array}\right], \quad B=\left[\begin{array}{cc}
\lambda_{2} & b \\
\bar{b} & -\lambda_{1}
\end{array}\right]
$$

(satisfying $\operatorname{Tr}(\rho A)=\operatorname{Tr}(\rho B)=0)$ Lemma 4 yields

$$
\begin{aligned}
F(\alpha)=\lambda_{1} \lambda_{2}|a-b|^{2} & \\
& +2\left(\lambda_{1}^{\alpha} \lambda_{2}^{1-\alpha}+\lambda_{1}^{1-\alpha} \lambda_{2}^{\alpha}\right)\left(1-\frac{1}{2}\left(\lambda_{1}^{\alpha} \lambda_{2}^{1-\alpha}+\lambda_{1}^{1-\alpha} \lambda_{2}^{\alpha}\right)\right)(\Im(a \bar{b}))^{2} .
\end{aligned}
$$


In particular, we have

$$
\begin{aligned}
F(1 / 2) & =\lambda_{1} \lambda_{2}|a-b|^{2}+4\left(\sqrt{\lambda_{1} \lambda_{2}}-\lambda_{1} \lambda_{2}\right)(\Im(a \bar{b}))^{2} \\
& =\lambda_{1} \lambda_{2}|a-b|^{2}+\left(1-\left(1-2 \sqrt{\lambda_{1} \lambda_{2}}\right)^{2}\right)(\Im(a \bar{b}))^{2}
\end{aligned}
$$

(which is non-negative due to $\left.\sqrt{\lambda_{1} \lambda_{2}} \leq\left(\lambda_{1}+\lambda_{2}\right) / 2=1 / 2\right)$. In [5, p.1574] this value of $F(1 / 2)$ was computed to confirm the conjecture (i.e., Theorem 5 with $\alpha=1 / 2$ ) for $2 \times 2$ matrices. On the other hand, (15) shows

$$
\left(\Im \operatorname{Corr}_{\rho}(A, B)\right)^{2}=\left(\lambda_{1}-\lambda_{2}\right)^{2}(\Im(a \bar{b}))^{2},
$$

and it is plain to see

$$
F(1 / 2)-\left(\Im \operatorname{Corr}_{\rho}(A, B)\right)^{2}=\lambda_{1} \lambda_{2}|a-b|^{2}+\left(4 \sqrt{\lambda_{1} \lambda_{2}}-1\right)(\Im(a \bar{b}))^{2}
$$

(thanks to $\lambda_{1}+\lambda_{2}=1$ ). This quantity cannot be non-negative for $\lambda_{1}$ sufficiently small (as long as $\Im(a \bar{b}) \neq 0$ ).

The situation cannot be improved even if $F(\alpha)(\geq F(1 / 2)), \alpha \in(0,1)$, is used. In the limit case $(\alpha=0$ or 1$)$ we have $\mathrm{I}(\rho, A, \alpha)=\mathrm{I}(\rho, B, \alpha)=\Re \operatorname{Corr}_{\rho}(A, B ; \alpha)=0$ so that

$$
F(0)=F(1)=\operatorname{Var}_{\rho} A \cdot \operatorname{Var}_{\rho} B-\frac{1}{4}\left|\operatorname{Cov}_{\rho}(A, B)+\operatorname{Cov}_{\rho}(B, A)\right|^{2} .
$$

We note that $F(1) \geq\left(\Im \operatorname{Corr}_{\rho}(A, B ; 1)\right)^{2}=0$ is obviously true while

$$
F(0) \geq\left(\Im \operatorname{Corr}_{\rho}(A, B ; 0)\right)^{2}=\frac{1}{4}|\operatorname{Tr}(\rho[A, B])|^{2}
$$

is nothing but Schrödinger's uncertainty principle (explained in Remark 2).

However,

$$
F(\alpha)-\left(\Im \operatorname{Corr}_{\rho}(A, B ; \alpha)\right)^{2} \nsupseteq 0
$$

can be easily checked for each $\alpha \in(0,1)$. In fact, similar computations as (15) show

$$
\Im \operatorname{Corr}_{\rho}(A, B ; \alpha)=\left(\lambda_{1}-\lambda_{1}^{\alpha} \lambda_{2}^{1-\alpha}+\lambda_{1}^{1-\alpha} \lambda_{2}^{\alpha}-\lambda_{2}\right) \Im(a \bar{b})
$$

so that with (16) we get

$$
\begin{aligned}
& F(\alpha)-\left(\Im \operatorname{Corr}_{\rho}(A, B ; \alpha)\right)^{2} \\
& =\lambda_{1} \lambda_{2}|a-b|^{2}+\left(2\left(\lambda_{1}^{\alpha} \lambda_{2}^{1-\alpha}+\lambda_{1}^{1-\alpha} \lambda_{2}^{\alpha}\right)\left(1-\frac{1}{2}\left(\lambda_{1}^{\alpha} \lambda_{2}^{1-\alpha}+\lambda_{1}^{1-\alpha} \lambda_{2}^{\alpha}\right)\right)\right. \\
& \left.-\left(\lambda_{1}-\lambda_{1}^{\alpha} \lambda_{2}^{1-\alpha}+\lambda_{1}^{1-\alpha} \lambda_{2}^{\alpha}-\lambda_{2}\right)^{2}\right)(\Im(a \bar{b}))^{2} .
\end{aligned}
$$

As $\lambda_{1} \searrow 0$ (and hence $\left.\lambda_{2}=1-\lambda_{1} \nearrow 1\right)$, this quantity tends to $-(\Im(a \bar{b}))^{2}$, showing that $F(\alpha)-\left(\Im \operatorname{Corr}_{\rho}(A, B ; \alpha)\right)^{2}$ cannot be always non-negative. 


\section{REFERENCES}

1. H. Araki and M. M. Yanase, Measurement of quantum mechanical operators, Phys. Rev., 200 (1960), 622-626.

2. J.-C. Bourin, Some inequalities for norms on matrices and operators, Linear Algebra Appl., 292 (1999), 139-154.

3. A. Connes and E. Størmer, Homogeneity of the state space of factors of type $\mathrm{III}_{1}$, J. Funct. Anal., 28 (1978), 187-196.

4. E. Lieb, Convex trace functions and the Wigner-Yanase-Dyson conjecture, Adv. Math. 11 (1973), 267-288.

5. S. Luo and Z. Zhang, An information charactrization of Schrödinger's uncertainty principle, J. Stat. Phys., 114 (2004), 1557-1576.

6. A. Wehrl, General properties of entropy, Rev. Modern Phys., 50 (1978), 221-260.

7. E. P. Wigner and M. M. Yanase, Information contents of distributions, Proc. Nat. Acad. Sci. USA, 49 (1963), 910-918.

Faculty of Mathematics, Kyushu University, Higashi-Ku, Fukuoka 812-8581, Japan 


\section{List of MHF Preprint Series, Kyushu University 21st Century COE Program Development of Dynamic Mathematics with High Functionality}

\section{MHF2003-1 Mitsuhiro T. NAKAO, Kouji HASHIMOTO \& Yoshitaka WATANABE}

A numerical method to verify the invertibility of linear elliptic operators with applications to nonlinear problems

MHF2003-2 Masahisa TABATA \& Daisuke TAGAMI

Error estimates of finite element methods for nonstationary thermal convection problems with temperature-dependent coefficients

MHF2003-3 Tomohiro ANDO, Sadanori KONISHI \& Seiya IMOTO

Adaptive learning machines for nonlinear classification and Bayesian information criteria

MHF2003-4 Kazuhiro YOKOYAMA

On systems of algebraic equations with parametric exponents

MHF2003-5 Masao ISHIKAWA \& Masato WAKAYAMA

Applications of Minor Summation Formulas III, Plücker relations, Lattice paths and Pfaffian identities

MHF2003-6 Atsushi SUZUKI \& Masahisa TABATA

Finite element matrices in congruent subdomains and their effective use for large-scale computations

MHF2003-7 Setsuo TANIGUCHI

Stochastic oscillatory integrals - asymptotic and exact expressions for quadratic phase functions -

MHF2003-8 Shoki MIYAMOTO \& Atsushi YOSHIKAWA

Computable sequences in the Sobolev spaces

MHF2003-9 Toru FUJII \& Takashi YANAGAWA

Wavelet based estimate for non-linear and non-stationary auto-regressive model

MHF2003-10 Atsushi YOSHIKAWA

Maple and wave-front tracking - an experiment

MHF2003-11 Masanobu KANEKO

On the local factor of the zeta function of quadratic orders

MHF2003-12 Hidefumi KAWASAKI

Conjugate-set game for a nonlinear programming problem 
MHF2004-1 Koji YONEMOTO \& Takashi YANAGAWA

Estimating the Lyapunov exponent from chaotic time series with dynamic noise

MHF2004-2 Rui YAMAGUCHI, Eiko TSUCHIYA \& Tomoyuki HIGUCHI

State space modeling approach to decompose daily sales of a restaurant into time-dependent multi-factors

MHF2004-3 Kenji KAJIWARA, Tetsu MASUDA, Masatoshi NOUMI, Yasuhiro OHTA \& Yasuhiko YAMADA

Cubic pencils and Painlevé Hamiltonians

MHF2004-4 Atsushi KAWAGUCHI, Koji YONEMOTO \& Takashi YANAGAWA

Estimating the correlation dimension from a chaotic system with dynamic noise

MHF2004-5 Atsushi KAWAGUCHI, Kentarou KITAMURA, Koji YONEMOTO, Takashi YANAGAWA \& Kiyofumi YUMOTO

Detection of auroral breakups using the correlation dimension

MHF2004-6 Ryo IKOTA, Masayasu MIMURA \& Tatsuyuki NAKAKI

A methodology for numerical simulations to a singular limit

MHF2004-7 Ryo IKOTA \& Eiji YANAGIDA

Stability of stationary interfaces of binary-tree type

MHF2004-8 Yuko ARAKI, Sadanori KONISHI \& Seiya IMOTO

Functional discriminant analysis for gene expression data via radial basis expansion

MHF2004-9 Kenji KAJIWARA, Tetsu MASUDA, Masatoshi NOUMI, Yasuhiro OHTA \& Yasuhiko YAMADA

Hypergeometric solutions to the $q$ - Painlevé equations

MHF2004-10 Raimundas VIDŪNAS

Expressions for values of the gamma function

MHF2004-11 Raimundas VIDŪNAS

Transformations of Gauss hypergeometric functions

MHF2004-12 Koji NAKAGAWA \& Masakazu SUZUKI

Mathematical knowledge browser

MHF2004-13 Ken-ichi MARUNO, Wen-Xiu MA \& Masayuki OIKAWA

Generalized Casorati determinant and Positon-Negaton-Type solutions of the Toda lattice equation

MHF2004-14 Nalini JOSHI, Kenji KAJIWARA \& Marta MAZZOCCO

Generating function associated with the determinant formula for the solutions of the Painlevé II equation 
MHF2004-15 Kouji HASHIMOTO, Ryohei ABE, Mitsuhiro T. NAKAO \& Yoshitaka WATANABE

Numerical verification methods of solutions for nonlinear singularly perturbed problem

MHF2004-16 Ken-ichi MARUNO \& Gino BIONDINI

Resonance and web structure in discrete soliton systems: the two-dimensional Toda lattice and its fully discrete and ultra-discrete versions

MHF2004-17 Ryuei NISHII \& Shinto EGUCHI

Supervised image classification in Markov random field models with Jeffreys divergence

MHF2004-18 Kouji HASHIMOTO, Kenta KOBAYASHI \& Mitsuhiro T. NAKAO

Numerical verification methods of solutions for the free boundary problem

MHF2004-19 Hiroki MASUDA

Ergodicity and exponential $\beta$-mixing bounds for a strong solution of Lévydriven stochastic differential equations

MHF2004-20 Setsuo TANIGUCHI

The Brownian sheet and the reflectionless potentials

MHF2004-21 Ryuei NISHII \& Shinto EGUCHI

Supervised image classification based on AdaBoost with contextual weak classifiers

MHF2004-22 Hideki KOSAKI

On intersections of domains of unbounded positive operators

MHF2004-23 Masahisa TABATA \& Shoichi FUJIMA

Robustness of a characteristic finite element scheme of second order in time increment

MHF2004-24 Ken-ichi MARUNO, Adrian ANKIEWICZ \& Nail AKHMEDIEV

Dissipative solitons of the discrete complex cubic-quintic Ginzburg-Landau equation

MHF2004-25 Raimundas VIDŪNAS

Degenerate Gauss hypergeometric functions

MHF2004-26 Ryo IKOTA

The boundedness of propagation speeds of disturbances for reaction-diffusion systems

MHF2004-27 Ryusuke KON

Convex dominates concave: an exclusion principle in discrete-time Kolmogorov systems 
MHF2004-28 Ryusuke KON

Multiple attractors in host-parasitoid interactions: coexistence and extinction

MHF2004-29 Kentaro IHARA, Masanobu KANEKO \& Don ZAGIER

Derivation and double shuffle relations for multiple zeta values

MHF2004-30 Shuichi INOKUCHI \& Yoshihiro MIZOGUCHI

Generalized partitioned quantum cellular automata and quantization of classical CA

MHF2005-1 Hideki KOSAKI

Matrix trace inequalities related to uncertainty principle 